\title{
Observaciones sobre los tratamientos de la Eclampsia
}

\author{
Por el Doctor Carlos R. Silva-Mojica \\ Ex-Jefe de Clínica Obstétrica.
}

No pretendo en este artículo exponer normas definitivas e invariables sino iniciar tan sólo un intercambio de ideas con los colegas acerca de la conducta más aconsejable y más racional en la eclampsia.

Tratamiento Médrco.-Siempre ha existido en nuestros colegas la tendencia de instituir inicialmente el tratamiento médico tanto en la preeclampsia grave como en la eclampsia convulsiva declarada. No hago mención ninguna de la preeclampsia leve, en la cual las medidas puramente médicas son indiscutibles. Manifiesto también mi total acuerdo con los métodos profilácticos de la eclampsia consistentes en un control prenatal estricto y riguroso. Si el éxito en el tratamiento de la preeclampsia consiste en impedir que aparezcan las convulsiones, no vacilo en afirmar en igual forma, que el éxito en el tratamiento de la eclampsia convulsiva consiste en impedir que aparezca el estado de coma.

¿Cómo evitar entonces la aparición de las convulsiones y del estado de coma? Esto es lo que vamos a tratar de puntualizar en la discusión de esta noche $\mathrm{y}$ en las investigaciones que sobre este punto deban necesariamente deducirse.

En la preeclampsia grave hay que tener en cuenta que el tratamiento médico no siempre es efectivo, sino que muchas veces, la insistencia en él puede llegar a ser fatal. A este propósito anoto dos casos desafortunados. El primero en el Instituto de Protección Materna e Infantil, en 1949, siendo yo Jefe de Clínica de uno de los servicios: se trataba de una multigestante, casi a término, con hipertensión convergente y sin edemas, cefalea y albuminuria. Fue sometida al tratamiento usual consistente en dieta, sulfato tomado e inyectado, fenobarbital y veratrum viridens. Veinte horas después presentó un ataque eclámptico, entró en coma y murió súbitamente sin que hubiera dado tiempo a alistar lo necesario para efectuar una cesárea tendiente a salvar la vida del feto ${ }^{1}$.

El segundo caso es mucho más reciente. Acaecido el 28 de octubre del presente en la Clínica Primero de Mayo. Paciente A. J. M. de 22 años, carnet No. 25266. Afiliada. Primigestante de 8 meses. Había sido controlada dos veces en el Dispensario Central del ICSS. Ingresó por fuerte cefalea iniciada unas 12 
horas antes. T. A. $220 / 160$ sin edemas. Feto vivo. Como primera medida se aplicó sulfato de magensia inyectado y en enema dextrosa al 20\%; fenobarbital y 3 unidades de Vergitril; pocos minutos después de ingerir el Vergitril presentó un ataque eclámptico; entró en coma; los ruidos fetales se hicieron negativos; la punción lumbar demostró líquido con sangre, signo de hemoriagia cerebral. $\mathrm{Se}$ la mantuvo con $\mathrm{O} 2$; morfina; sulfato inyectado. Falleció cuatro horas después ${ }^{2}$.

En estos dos casos el tratamiento médico de observación fue inefectivo; hizo perder un tiempo precioso, durante el cual, apreciada la gravedad de las condiciones se hubiera podido decidir desde un principio la operación cesárea con buenos resultados para la madre y para el hijo. Esta es mi impresión.

Se pudiera decir que es asunto de criterio médico el seguir tal o cual conducta ante determinado caso. Pero todos hemos visto casos en que es difícil juzgar esa extrema gravedad y determinar si es posible esperar un tiempo prudencial de observación bajo un tratamiento médico racional. Se ven casos de suma gravedad, como los citados antes, ceder con la aplicación de esos tratamientos y casos más leves en los que ha sido necesario recurrir a la inducción y a la cesárea en vista de persistencia o agravación de los síntomas. $\mathrm{Al}$ respecto, me permito citar un caso: Paciente G. F. B. de 27 años. Grávida IV de 8 meses. Feto vivo. Ingresa a la Clínica Primero de Mayo el 21 de noviembre de 1953, con cefaela, epigastralgia, visión de fosfenos; sin edemas; TA 170/120. Es decir, un caso de preeclampsia grave. Sin embargo ha reaccionado en buena forma al tratamiento médico. En las primeras 24 horas se trató con dieta, reposo, sulfato de magnesia inyectado y fenobarbital. Como el cuadro no variara se suspendieron estas drogas y se empezó a dar vergitril en dosis ascendentes, de 3 a 6 unidades. Al quinto día la TA estaba en 110/60. No había albuminuria. Se suspendió el vergitril y se conservó la dieta y el reposo solamente. La TA no volvió a subir y desaparecieron los fenómenos anotados, lo cual indicaba que la toxemia había sido yugulada ${ }^{2}$.

Según lo expuesto en la Primera Convención de Obstetricia y Ginecología, prima entre nosotros un criterio médico en el tratamiento de la preeclampsia. Las estadísticas de Corral, Pérez y Gaitán demuestran la mayor frecuencia del tratamiento médico: $74,8 \%$; en cambio un $4,6 \%$ para la cesárea; $10,2 \%$ para el forceps y $10,2 \%$ sin dato 3 .

El Profesor Acosta por su parte, recalcó sobre la importancia de las medidas higiénicas y dietéticas y sobre el buen efecto de veratrum usado convenientemente .

Respecto de los resultados de los antihistamínicos en la toxemia gravídica no se puede deducir conclusión de mayor valor, debido al reducido número de casos estudiado. Anoto tan sólo, como lo dice el Profesor Peralta, que en la intoxicación gravídica no tienen tanta eficacia terapéutica como en la intoxicación histamínica ${ }^{5}$.

Por mi parte, al tratar sobre la mortalidad materna, anoté lo siguiente: "No insistir neciamente con un tratamiento médico, por intenso y bien dirigido que sea, si los síntomas clínicos indican una agravación progresiva de la paciente, sino 
acudir a las medidas quirúrgicas cuanto antes, sobre todo si ha habido fracaso de inducción. Se debe tener presente que la insistencia en un tratamiento médico, en caso de agravación de la eclampsia, conduce al riesgo de perder dos vidas; en cambio el acudir oportunamente a la vía quirúrgica asegura por lo menos una vida"'s.

Tratamiento Quirúrgico de la Eclampsia.-Desconozco los datos pre sentados por el Instituto de Protección Materna e Infantil en la Convención, respecto de la cesárea en las toxemias gravídicas. En todo caso me interesa conocer estos datos y estos resultados. A propósito anoto que durante mi jefatura de clínica (1947-1951) en esa institución, en un total de 68 cesáreas, en 6 casos se hizo esta intervención por eclampsia, lo cual da un $8,8 \%$, con resultados sa. tisfactorios para la madre y el niño. Igualmente observé que se acudió a la vía quirúrgica en vista del fracaso del tratamiento médico y del empleo del veratrum viridens en 3 de ellos ${ }^{7}$.

Respecto de la Clínica Primero de Mayo es difícil anotar datos exactos acerca del tratamiento de la preeclampsia y de la eclampsia, debido a la ausencia casi absoluta de estadística. Deduzco en todo caso por las juntas médicas a que asisto y por referencias de los colegas, que allí la eclampsia se resuelve por cesárea en el 95\% de los casos. Es difícil anotar los resultados del tratamiento médico porque de ello a veces no queda constancia alguna, o si queda, es muy laborioso hallar los datos en los libros de juntas o en historias hechas sin método estadístico ninguno.

El dato más diciente respecto del tratamiento quirúrgico de la eclampsia es el que arrojan los records de anestesia de la sala de cirugía de la clínica. Aunque el número de cesáreas por eclampsia es escaso, los resultados sí son muy ilustrativos:

Número de cesáreas por eclampsia, 17.

Estado de las pacientes: comatoso, 1 caso; semicomatoso, 8 casos; regular estado, 8 casos.

Mortinatalidad, 0 casos.

Hubo en total 18 niños vivos (un caso de gemelos), de los cuales 3 fueron prematuros.

Mortalidad materna, 2 casos $(11,7 \%)$.

Una paciente que se encontraba en estado semicomatoso y otra en coma, casi en estado agónico fue operada para obtener el niño vivo, como así sucedió.

Anestesia empleada: Raquia-anestesia, 11 casos; Ciclo O2, 5 casos; O2 (paciente agónica), 1 caso.

Anoto que las enférmas sobrevivientes salieron en buen estado de la clinica sin que hubiesen manifestado por efecto de la escurocaína o del ciclopropano, agravación de sụs lesiones renales, vasculares y hepáticas, o a lo menos no hallé constancia de esto ${ }^{8}$.

Los resultados favorables de este intervencionismo saltan a la vista al ser comparados con los datos del IPMI (Instituto de Protección Materno-Infantil) donde las conductas son más conservadoras". 
ICSS - Indice de mortalidad materna, 1,6\% eclampsia, $0,7 \%$ \%

IPMI - Indice de mortalidad materna, 8,4\% ${ }_{n o}$; Indice de mortalidad por eclampsia, 1,6\%

En la misma clínica Ofman encontró en 204 cesáreas, que 25 fueron efectuadas con indicación de toxemia gravídica, lo cual da un porcentaje para esta indicación del $12 \%$. Martínez-Sáenz anota también un porcentaje de 12,2\%\%.

Aplicación de la Inducción en la Toxemia.-Es conveniente, ante el fracaso del tratamiento médico de las toxemias, hacer un intento de inducción antes de acudir a la vía quirúrgica. Aunque algunos desaconsejan el empleo de acitócicos para este fin por el peligro de agravar la toxemia ${ }^{3}$ hay experiencias $y$ literatura nacional y extranjera sobre la inocuidad de este método ${ }^{10}$. Aunque mi experiencia en este punto es muy limitada, tengo la impresión de que puede ser usado el pitocín, con las debidas precauciones, ya que está desprovisto del factor vasopresor de la hipófisis, en dosis de 3 a 5 u. en 500 c. c. de dextrosa al 10 o al $20 \%$ en agua. Así es como lo he usado y como tengo noticia de que lo usan los colegas con la aplicación simultánea de sedantes e hipotensores, como el fenobarbital y el sulfato de magnesia.

Opiniones en Contra de la Cesárea.-Entre especialistas americanos e ingleses prevalece hoy día una marcada tendencia a tratar la toxemia en forma puramente médica. Posner, citado por Fernández-Bastidas, anota como un hecho generalmente admitido, que no se debe practicar la cesárea en caso de toxemia sino cuando existe una indicación obstétrica, o cuando el cuello no permite un parto inmediato por vía vaginal, puesto que la cesárea no garantiza la cesación inmediata de las convulsiones ${ }^{11}$.

Greenhill admite la cesárea en la primigrávida con feto viable que no ha respondido a un tratamiento médico bien dirigido. En cambio la contraindica en la eclampsia, salvo contados casos ${ }^{12}$.

Tengo la impresión de que en otras latitudes sí sea efectivo el tratamiento médico de la toxemia por razones de clima, de raza, de estado nutricional, de mejor control prenatal y de recursos clínicos más perfectos. Todo esto hace que el organismo de la gestante responda en forma satisfactoria a los diversos fármacos aplicados. Pero entre nosotros, dadas nuestras condiciones totalmente diferentes, creo que al apartarnos de las medidas quirúrgicas, no haríamos otra cosa sino aumentar los índices de mortalidad materna y de mortinatalidad. Pero en todo caso se hace necesario entre nosotros una revisión fundamental de los sistemas de tratamiento médico y un detenido estudio de sus resultados.

Consideraciones Sobre el Hyo de la Toxémica.-Gutiérrez, Sabogal y Sánchez anotan una elevada mortalidad fetal en la toxemia gravídica de $22,6 \%$. Presentan como causas principales la intoxicación del feto inherente a la toxemia, la prematurez y la desnutrición de las pacientes hospitalarias. Consideran que en relación al feto es más ventajosa la conducta intervencionista ${ }^{13}$.

Plata-Rueda hace notar que el mayor intervencionismo sobre la toxemia ha sido uno de los factores de disminución de mortinatos en la toxemia, al lado 
de un mayor control prenatal, de una mayor educación popular y de una atención más eficiente en IPMI ${ }^{14}$.

\section{SUMARIO}

Puntualizo en la siguiente forma los métodos de prevención y tratamiento de la toxemia gravídica.

$1^{9}$-Mejorar las condiciones de vida de las gestantes (alimentación, vivienda, condiciones generales de higiene).

$2^{\circ}$-Control prenatal estricto.

$3^{\circ}$ - Campaña de educación popular sobre los cuidados del embarazo.

$4^{\circ}-$ Dieta y drogas apropiadas cuando aparezca la toxemia y hospitalización cuando el caso lo requiera.

$5^{\circ}-$ Tratamiento médico bien llevado en la preeclampsia.

$6^{\circ}$-Inducción si en la preeclampsia no surte efecto el tratamiento médico.

$7^{\circ}$-Cesárea, en casos de preeclampsia grave o de eclampsia, tanto más si ésta en su estado de preeclampsia no respondió a tratamiento médico.

$8^{\circ}$-Forceps en todos los casos de preeclampsia y eclampsia cuando las condiciones de dilatación y encajamiento lo permitan.

90-Revisión de nuestros sistemas de tratamiento médico y un mejor estudio de sus resultados.

\section{BIBLIOGRAFIA}

1.-Estadística del IPMI de Bogotá. 1949. Servicio del Profesor Mojica.

2.-Historias Clínicas de la Clínica Primero de Mayo. ICSS. Bogotá, 1953.

3.-Corral, Pérez, Gaitán.--Tratamiento de la Preeclampsia. Primera Convención Nacional de Obstetricia y Ginecología, 1953. Inédito.

4.-Acosta-Villaveces.-Tratamiento de la Eclampsia. Primera Convención Nacional de Obstetricia y Ginecología, 1953. Inédito.

5.-Peralta R.-Antihistamínicos en el Tratamiento de las Toxemias Gravídicas. Primera Convención Nacional de Obstetricia y Ginecología, 1953. Inédito.

7.-Silva-Mojica.-Comentarios Clínicos y Estadísticos Sobre Operación Cesárea. Trabajo presentado en la Sociedad Colombiana de Obstetricia y Ginecología en marzo de 1950.

8. -Records anestésicos de la Clínica Primero de Mayo. 1953, hasta diciembre 19.

9. - Ofman.-Consideraciones Sobre Cesáreas Practicadas en el ICSS. Rev. Col. de Obs. y Gin., Vol. V, pág. 44, 1953.

10. -Rev. Col. de Obs. y Gin., Vol, V, págs. 21, 22, 66. 1953.

11.-Fernández-Bastidas.-Rev. Col. de Obst. y Gin. 5: 22.

12.-Greenhill.-The Sur. Cl. of North America. 1953: 33, 87. Citado por Rev. Col. de Obst. $y_{\text {. Gin. Vol. V, No. } 1 .}$

13.-Gutiérrez, Sabogal, Sánchez.-La Mortalidad Fetal en las Toxemias Gravídicas. Primera Convención Nacional de Obstetricia y Ginecología. 1953. Inédito.

14.-Plata-Rueda E.-Consideraciones del Recién Nacido de la Toxémica. Primera Convención Nacional de Obstetricia y Ginecología. 1953. Inédito.

NOTA: Próximamente serán publicadas por la Sociedad Colombiana de Obstetricia y Ginecología las "Memorias de la Primera Convención Nacional de Obstetricia y Ginecología” con todos los trabajos que allí se presentaron. 